\title{
Hybrid based Energy Efficient Cluster Head Selection using Camel Series Elephant Herding Optimization Algorithm in WSN
}

\author{
N. Lavanya ${ }^{1}$, T. Shankar ${ }^{2}$ \\ Department of Communication Engineering \\ School of Electronics Engineering \\ Vellore Institute of Technology, Vellore, Tamil Nadu, India
}

\begin{abstract}
The rapid growth in wireless technology is enabling a variety of advances in wireless sensor networks (WSNs). By providing the sensing capabilities and efficient wireless communication, WSNs are becoming important factor in day to day life. WSNs have many commercial, industrial and telecommunication applications. Maximizing network lifespan is a primary objective in wireless sensor networks as the sensor nodes are powered by a non-rechargeable battery. The main challenges in wireless sensor networks (WSNs) are area of coverage, network's lifetime and aggregating. Balanced node establishment (clustering) is the foremost strategy for extending the entire network's lifetime by aggregating the sensed information at the head of the cluster. The recent research trend suggests Meta-heuristic algorithms for the intelligent selection of ideal Cluster Heads (CHs). The existing Cluster Head Selection (CHS) algorithm suffers from the inconsistent trade-offs between exploration - exploitation and global best examine constraints. In this research, a novel Camel series Elephant Herding Optimization (CSEHO) algorithm is proposed to enhance the random occurrences of Camel algorithm by the Elephant Herding Optimization algorithm for optimal CHS. The Camel algorithm imitates the itinerant actions of a camel in the desert for the scavenging procedure. The visibility monitoring condition of the camel algorithm improves the efficiency of exploitation, whereas the exploration inefficiency of a Camel algorithm is compensated optimally by the Elephant Herding Optimization operator (Clan and separator). The superior performance of the proposed CSEHO algorithm is validated by comparing its performance with various other existing CHS algorithms. The overall attainment of the offered CSEHO algorithm is $21.01 \%$, $31.21 \%, 44.08 \%, 67.51 \%$, and $85.66 \%$, better than that of EHO, CA, PSO, LEACH, and DT, respectively.
\end{abstract}

Keywords-Camel algorithm; Cluster Head Selection; Elephant Herding Optimization; meta-heuristic algorithm; network lifespan; wireless sensor network

\section{INTRODUCTION}

A sensor network incorporates multiple minimal prices and low-battery sensor nodes. Every sensor nodes are situated in a specific zone and typically install a remote system by the method of self-sorting out. The sensor nodes can investigate typically at some of the extraordinary and critical circumscribe that individuals cannot handle [1]. Due to various complex factors, information communication between nodes in an effective manner is almost impossible. An energetic clustering is a well-known system to increase the effectiveness of sensed data transmission. The fundamental concept of clustering is to boundaries around the nodes of the active sensor into separate groups and chooses leaders for all groups. The groups are known as clusters while group leaders are known as Cluster Heads (CHs) of the groups to perform data communication [2]. In such condition, electing the $\mathrm{CH}$ under several constraints such as less amount of energy consumption, distance, delay and so on is the vital character of every clustering algorithm. In the real-world, $\mathrm{CH}$ renewed through different iterations contributes towards the finest attainment. The individual cluster comprises a $\mathrm{CH}$ with finite cluster associates. The responsibility of the $\mathrm{CH}$ is that it should organize each and every node existing in the cluster.

Information communicated to the sink node (BS) from every sensor node over an energetic $\mathrm{CH}$ is an important experiment exhibited for the routing technique [3]. Best $\mathrm{CH}$ election structure results in reduction of transmission energy, transmission time, transmission distance and so on. This paper besides deliberates some energy-efficient clustering algorithm in a wireless sensor network and introduces an optimal metaheuristic algorithm for efficient $\mathrm{CH}$ election in WSN. Among the several meta-heuristic optimization techniques, maximum of them were implemented to achieve the best $\mathrm{CH}$ election in WSN. Meta-heuristics are generally inspired by physical phenomena, animal behaviour, or evolutionary concepts [4]. The ease enables a researcher to simulate various natural ideas, suggest fresh algorithms, integrate many meta-heuristics, or enhance present meta-heuristics. This further, helps other researchers to rapidly know and apply meta-heuristics to their issues. In general, meta-heuristics may be categorized into two foremost groups: single-value established and a set solution (population) established. In the first case, the search method begins with one candidate solution. In the course of iterations, this single candidate solution is then enhanced. However, population-based meta-heuristics use a set of solutions (population) to conduct optimization. In this technique, the exploration procedure begins with an arbitrary primary population (set solutions), and during the progression of rounds, this population is improved. Based on the shared information, population-based meta-heuristics can suddenly jump towards a better optimal location and avoid local optimal location and superior exploration than the single valueestablished algorithms [4]. The existing meta-heuristic method 
frequently suffers from numerous problems like an exploration - exploitation tradeoff, moderate convergence speed and in addition it is not as much of ability in considering the multiobjective. This inspires us to obtain a global search with fast convergence; a Camel series Elephant Herding Optimization (CSEHO) algorithm is proposed to select an energy efficient cluster head selection in WSN.

Camel algorithm (CA) is the latest renowned optimizer unit that works on the itinerant performance of a camel in the desert for scavenging procedures. Camels have the ability to identify the optimal food source location present in the desert and also share the identified optimal food location to other camels that are present in the visibility region [5]. Hence for exploitation, a camel algorithm is employed. For a further broader search, Elephant Herding Optimization (EHO) is employed in the proposed work. EHO algorithm is constructed on the assembling attitude of elephant groups under the supervision of an elephant queen (matriarch). The main aim of the CSEHO is to prolong the lifespan of the modelled WSN by employing EHO for exploration and CA for exploitation. This proposed CSEHO technique combines the advantages of both CA and EHO, bringing the equilibrium among the exploitation and exploration stages of optimization, resulting in better performance of the network.

The remaining portions of the research article are arranged in a manner listed as Section 2 deliberates the associated works proposed in the literature review; Section 3 elaborates on the method offered; Section 4 presents the simulation results and discussion; Section 5 presents the conclusion for the work implemented and the scope to extend further.

\section{LITERATURE REVIEW}

Heinzelman, W.B. et al. (2002) [3,6] proposed a centralized protocol called LEACH-C, in this the responsibility of the $\mathrm{CH}$ election and the delivery of info into the sensor network are maintained by the Sink node. Because of the fact that the steady-state level is entirely implemented at the Sink node, sensor nodes are not affected by overheads at the period of cluster establishment. The drawback of the LEACH protocol is that it performs an arbitrary election of $\mathrm{CHs}$, which election inefficient $\mathrm{CHs}$ and thus makes to extremely unproductive lifespan and energy absorbent by the network. Younis, O., \& Fahmy, S. (2004) [7] proposed a Hybrid Energy Efficient Distributed (HEED) clustering algorithm. The residual energy of nodes and intra communication costs are the two main parameters that have been used in this approach for the selection of $\mathrm{CH}$ among sensor nodes. HEED provides even distribution of $\mathrm{CHs}$, and the chances for two nodes within the same communication range can be selected as $\mathrm{CHs}$ is avoided. The major drawback found in this method is the overhead caused by energy dissipation. To extend the life span of a WSN, nodes are determined to sleep by the Sleep Scheduling methodology. Basically, Sensor nodes are very lucky to sleep consuming Sleep Scheduling mechanisms structured by the base station to increase energy efficient management [8].

In recent years several investigators have established natural inspiration based optimization techniques that imitate certain genetic actions or physical occurrences. Hussain et al. investigated smart techniques using a genetic algorithm (GA) for cluster construction and control. The optimal cluster head selection process is carried out at the base station using GA [9]. The GA is sensitive to the primary population and it may converge at local minima. The authors in $[10,11]$ have recommended a PSO algorithm that is founded based on the natural activities of a group of birds. The PSO uses the cumulative effect of personal best and global best to generate a new direction for each particle in the search region. The gravitational search algorithm for the node localization in WSN was presented in [12]. Hence it needs an improvement in energy consumption and stability of localization method. In [13], enhanced PSO based clustering energy optimization algorithm (EPSO-CEO) in WSN for $\mathrm{CH}$ selection is done through PSO algorithm and concerning the less power consumption in WSN with high delay.

In [14], an optimal based clustering technique using artificial bee colony (ABC) algorithm and factional calculus were presented for the purpose of maximizing the network energy and lifetime. The cluster heads are selected optimally using the ABC algorithm. In [15], Dynamic clustering based routing protocol with the generalized ACO algorithm was presented to increase the lifespan of SNs by energy constraints. The protocol consumes around $25 \%$ of the whole energy consumption of the network for data transmission. The overhead of this approach is relatively high and so the network lifetime is relatively short. In [16], Harmony search algorithm (HSA) based CH selection algorithm was offered to solve the wide range of NP hard problem with effective mapping and fitness function by means of energy, distance and node degree. This technique doesn't consider fault tolerance and delay.

Yang [17] proposed a flower pollination algorithm initiated on the development of flower pollination of blossoming flowers seeing the biological reproduction cycle. The researcher focuses on the specialty of sunflowers' motion in the exploration for the best orientation in the direction of the sun. The random fertilization is considered between the marginal distances of sunflower $i$ and $i+1$. In general, millions of pollen gametes are frequently released by every flower patch. For ease, we consider that every sunflower generates only one game of pollen and reproduces individually. The authors in [18] suggested a non-dominated fashion of cluster head selection using a multi-objective evolutionary algorithm (MOEA) called NSGA-II for extending the lifespan of the WSN. The energy consumption function is analysed to improve the life period of the WSN. The network loss the node at the prime stage is a major limitation of this method. The authors in $[19,20]$ suggested a routing method to incorporate cluster establishment, and multipath routing through reduced energy consumption and routing overheads.

Several research undertakings the hybridization of two optimization algorithms to overcome the disadvantages of the algorithms working independently. Shankar, T. et al [21] suggested a hybrid HSA-PSO bring exploration-exploitation trade-off in the optimization problem of cluster head selection in WSNs. This method combines extraordinary examine efficiency of HSA and dynamic nature of PSO, to produce an improved performance. The performance in terms of the first dead node is good. However, the last dead node round number needs improvement. Lavanya, N. et al. [22] suggested a hybrid 
squirrel harmony search algorithm for extending the lifespan of the WSN. The Squirrel search algorithm is established by engaging the method of improving the positions of the squirrels with respect to predator presence probability and seasonal monitoring condition. The stability among the exploitation and the exploration is achieved by the gliding constant. The performance in terms of the last dead node is good. However, the residual energy needs improvement.

From the literature, the single optimization methods either excel in the exploration phase of optimization or the exploitation phase, but lags in balance among the exploration and exploitation phases. Hence the proposed work presents the hybridization of EHO algorithm for replacing the random search of a camel algorithm to balancing exploration and exploitation, thereby providing better performance in the optimal selection of CHs in WSN.

\section{RESEARCH METHODOLOGY}

\section{A. Wireless Sensor Network Model}

In this research, the overall transmission energy of the SN is considered by free space network model as given in equation (1-2). It is assumed that the separation distance between the receiver unit and transmitter unit is $\mathrm{x}$, the information length is $\mathrm{I}$, and a group of sensor nodes are scattered in a rectangle fashion [18].

$E_{T x}(I, x)=\left\{\begin{array}{c}I_{e c}+I_{f_{f s} x^{2}, x<x_{0}} \\ I E_{e c}+I_{a m p} x^{4}, x \geq x_{0}\end{array}\right.$

$\mathrm{E}_{\mathrm{Rx}}(\mathrm{I})=\mathrm{IE}_{\mathrm{ec}}$

where $E_{e c}$ is the electronics energy; $E_{R x}$ signifies the energy used up by the receiver for the communication of bit length I, and $E_{T x}$ signifies the energy used up by the transmitter for the same information. In the molded WSN, n number of sensors is arbitrarily settled in a field of area ' $\mathrm{M} \mathrm{x} \mathrm{N}$ ' $\mathrm{m}^{2}$. For computing the optimal ' $\mathrm{k}$ ' Cluster head, the following fitness function is employed [13]:

$\mathrm{f}_{\text {obj }}=\varepsilon \times \mathrm{f}_{1}+(1-\varepsilon) \times \mathrm{f}_{2}$

where $f_{1}=\max _{k}\left\{\sum_{\forall,{\text { node }, i \in C_{k}}_{k}} \frac{\left.\text { (node }_{i}, \mathrm{CH}_{k}\right)}{\| \text { (luster }_{k} \|}\right\}$ and $f_{2}=$ $\frac{\sum_{i=1}^{N} E\left(\text { node }_{i}\right)}{\sum_{j=1}^{k} E\left(\mathrm{CH}_{j}\right)}$ are distance and energy optimization fitness function; $\varepsilon \in[0,1]$;

\section{B. Camel Algorithm}

Mohammed et al. (2016) proposed a meta-heuristic optimization method which imitates the itinerant performance of a camel in the desert for scavenging process. The Camel familiarizes itself with the desert to tolerate scarcity of water and high temperature for extended periods of time so that it can survive with limited or insufficient of existing food resources. Over the snowy and cold times, the camel can manage themselves without intake for more than a few months. The camels spread through an influenced area in search of the food spot. When a certain camel enters a food resource, it interacts with the other members of the caravan to follow that commercial food resource. The caravan members can adjust their route until they reaches the optimal food resource.
Because of the presence of sand dunes, the camel may fail to follow the optimal path to the food resource and start searching alternate food sources in a random location. Other camels may identify improved food locations during this trip, so other camels can change their route based on the received information about the new location. The cycle goes on until the camels grasp an oasis.

\section{Camel Algorithm Implementation}

Camel algorithm starts when random position of camels begins scavenging. The sand dunes and distance affects the camel's visibility. The following steps are implemented in the camel algorithm:

Step 1: Random initialization: In a desert, it is considered that there is $\mathrm{N}$ number of camels scattered randomly to identify the optimal food source and the initial position of all the camels is given in the following equation:

$x_{i}=x_{\min }+r d \times\left(x_{\max }-x_{\min }\right)$

where $x_{\min }$ and $x_{\max }$ are minimum and maximum of search bounds correspondingly; whereas rd refers to arbitrarily created value well-defined in the period $[0,1]$.

Step 2: Fitness assessment: Based on the initial energy and position the fitness standards of every camel are obtained, consequently they are arranged in an ascending fashion. The lowest fitness is declared as global optimal solution (global_best).

Step 3: The camel endurance $(E)$ : The travelling distance particularly impacts the camels' energy and it reduces the camels' endurance directly. The multiple camels travel along different paths toward the optimal solution and meeting the different values of distance resultant in dissimilar endurance value for each individual. The distance $\mathrm{D}$ of camel $i$ at the iteration $\mathrm{t}$ is given as follows:

$D_{i}(t)=D_{\text {min }}+r d \times\left(D_{\text {max }}-D_{\text {min }}\right)$

where $D_{\min }$ and $D_{\max }$ are minimum and maximum of travelling distance; whereas rd refers to arbitrarily created value well-defined in the period $[0,1]$.

It is suggested that the impact of distance on camel endurance $\mathrm{E}$ as given in the following equation:

$E_{i}(t)=1-\frac{\left(D_{i}(t)-D_{\min }\right)}{\left(D_{\max }-D_{\min }\right)}$

where $D_{i}(t)$ refers to initial distance of the camel. The equation (6) shows that, the distance is inversely related to the camel endurance.

Step 4: New location generation: The sand dunes may fail to update the camel location towards an optimal food resource identified by other camels. The searching movement of the camels can be modelled accurately from the following two Scenarios:

Scenario 1: The visibility of the camel is greater than the visibility threshold. The new location updates function as given in the following equation:

$x_{i}(t+1)=x_{i}(t)+E_{i}(t) \times\left(x_{\text {global_best }}-x_{i}(t)\right)$ 
where $x_{\text {global_best }}$ and $x_{i}(t)$ are refers to global best and initial position, $E_{i}(t)$ is the endurance.

Scenario 2: The visibility of the camel is lesser than the visibility threshold. On bearing this condition, the random updating of the camels is accomplished in the following equation (8):

$x_{i}(t+1)=x_{\text {min }}+r d \times\left(x_{\text {max }}-x_{\text {min }}\right)$

where $x_{\min }$ and $x_{\max }$ are minimum and maximum of search bounds correspondingly; whereas rd refers to arbitrarily created value well-defined in the period $[0,1]$.

Step 5: New locations fitness assessment: Evaluate the fitness for all the new locations and assign the new best location as the global best if it is improved than the existing global best.

Step 6: Stopping Criterion: The procedure is recurrently executed for a defined number of search iterations. The pseudocode of camel algorithm for header node selection as follows:

Pseudocode of Camel Algorithm:

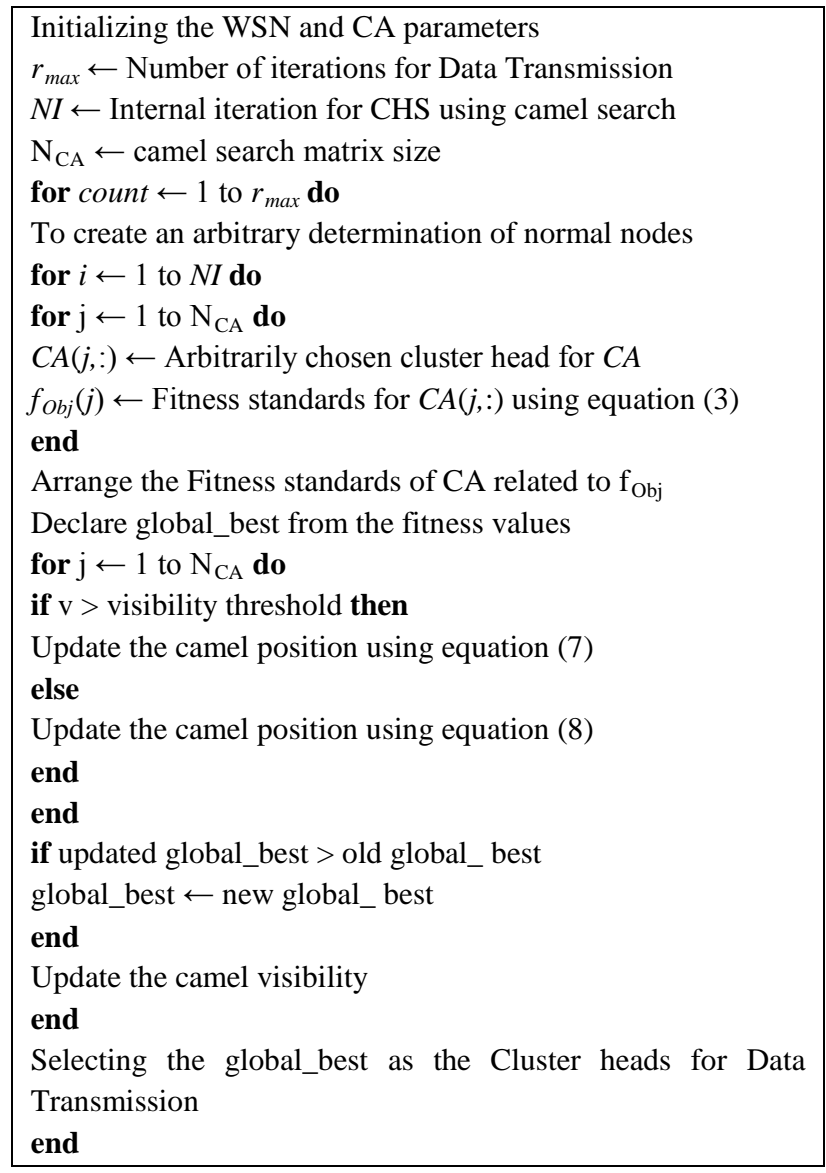

\section{Elephant Herding Optimization (EHO) Algorithm}

G.G.Wang et al. [23] proposed a new meta-heuristic optimization method which imitates the assembling attitude of elephant groups represented as the Elephant Herding Optimization (EHO) algorithm. The elephant population is divided into a small number of subgroups called as a clan under the supervision of an elephant queen (matriarch). When the elephant king (male elephant) maturing up, he would like to stay away from the family and contact with the clan at low frequency. The supervision and stay away nature of the elephant builds the EHO algorithm into two operatives: clan updating (supervising) operative and separating (staying away) operative. The elephants are updated using their present location and matriarch through clan updating operative, and the separating operative is then executed.

\section{E. Mathematical Model of Elephant Herding Optimization (EHO) Algorithm}

The process of EHO is initialized by the leadership activities of elephant group. In woodland, it is considered that there is $\mathrm{P}$ number of elephants scattered randomly and this elephant population $(\mathrm{P})$ is separated into $\mathrm{N}$ number of clans. Each clan may update the position under two operatives [24]. The elephant clan updating behaviour is represented as follows.

Clan operative: The elephants survive in an organized manner under the headship of an elephant queen (matriarch) in each set. As a result, for all the elephants in clan cni, its update location is determined by the elephant queen (matriarch) ci. The updated locations of elephant $j$ in clan cni as given in the subsequent equation:

$x_{n e w, C n_{i}, j}=x_{C n_{i}, j}+\varepsilon \times r d \times\left(x_{\text {best }, C n_{i}}-x_{C_{n i}, j}\right)$

where $x_{n e w, C n_{i}, j}, x_{C n_{i}, j}$ and $x_{b e s t, C n_{i}}$ are recently updated location, past location and fittest location of elephant $j$ in clan $C n_{i}$, whereas scaling parameter $(\varepsilon)$ and rd refer to arbitrarily created values well-defined in the period $[0,1]$.

Updating the fittest elephant: Based on the clan members, the elephant queen (fittest elephant) can update its own location in following equation:

$x_{\text {new }, C_{i}, j}=\delta \times\left(x_{\text {center }, C n_{i}}\right)$

where $\delta$ refer to arbitrarily created values well-defined in the period [0,1], whereas $x_{\text {center, } C_{n i}}$ represents the midpoint of clan $c_{n i}$. It is obtained by equation (11):

$x_{\text {center }, C n_{i}, d}=\frac{1}{n_{C n_{i}}} \times \sum_{j=1}^{n_{C n_{i}}} x_{C n_{i}}, j, d$

Separating operative: In elephant clusters, the male elephant will live separately from the clan when they reach maturity. This separating procedure offering the separating operator presenting each generation as the elephant individuals with the poorest fitness, which is realized as follows:

$x_{{\text {worst }, C n_{i}}}=x_{\text {min }}+\left(x_{\max }-x_{\text {min }}+1\right) \times r d$

where $r d \in[0,1] ; x_{\min }$ and $x_{\max }$ are minimum and maximum of search bounds; whereas $x_{\text {worst, } \mathrm{Cn}_{i}}$ refers to the lowest fitness in clan $c_{n i}$.

New location fitness assessment: Evaluate the fitness for all the new locations and assign the new best location as the global best if it is improved than the existing global best. 
Stopping Criterion: The procedure is recurrently executed for a defined number of search iterations. The pseudocode of EHO algorithm for header node selection as follows:

Pseudocode of EHO Optimization

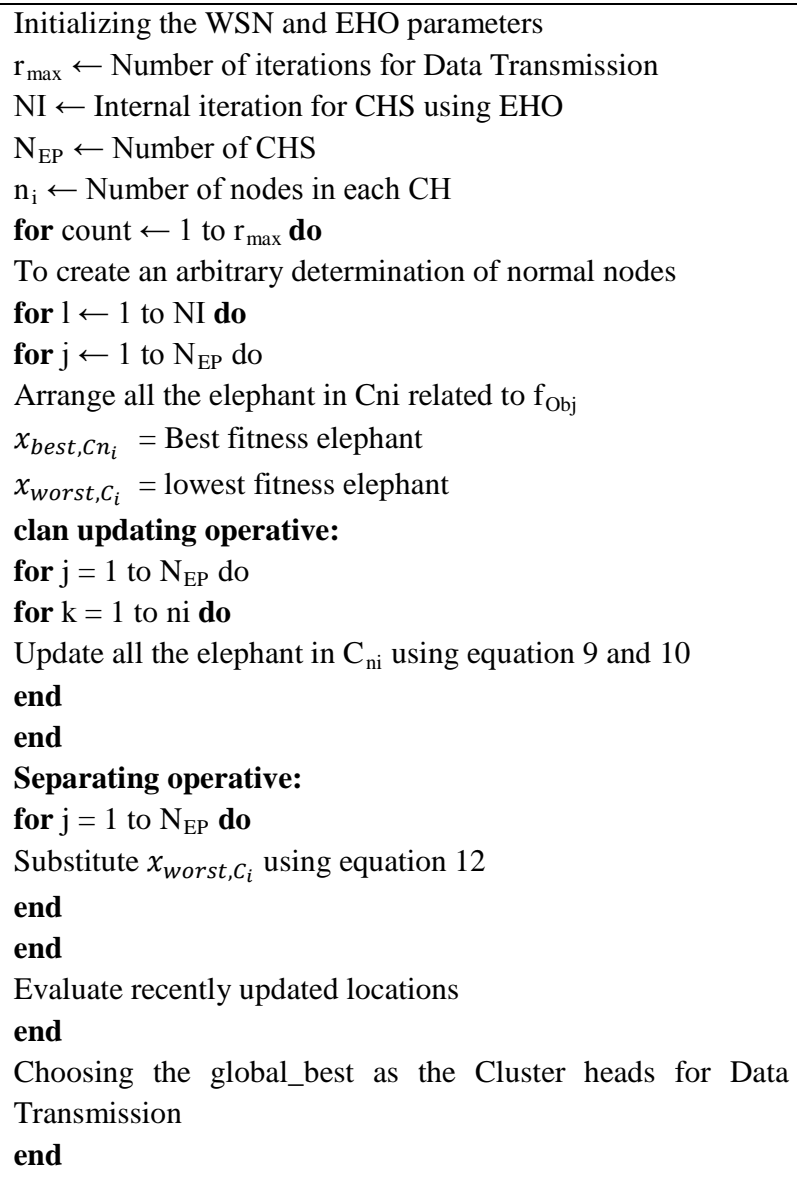

F. Proposed HCSEHO Algorithm for Optimal Cluster Head Selection

The proposed camel series elephant herding optimization algorithm integrates the general attributes of camel algorithm and elephant herding optimization algorithm. The camel visibility is directly related to distance and sand dunes. The designed objective of camel algorithm is to identify the optimal location for all the nodes based on the camel visibility. The visibility monitoring condition is established in camel algorithm to exclude the offered technique from being bound to the results of local best and provide an accurate approach towards reaching the optimal result. Even though the visibility monitoring condition is established in camel algorithm, still it faces the high dimensionality limitation. In order to achieve the exploration and exploitation with high search efficiency in high dimensionality, the randomly searching process of the camel algorithms is switched by EHO algorithm. The flow figure of the offered HCSEHO scheme is presented in the Fig. 1.

In HCSEHO algorithm, employing camel algorithm allows the camels to travel around from one region to other region by modifying the positions and visibility at the end of every round. The energetic performance of Camel algorithm decides to find optimal cluster heads (CHs) present in the n number of clusters with high search efficiency of EHO algorithm. The frame work of the proposed CSEHO algorithm is to perform energy proficient transmission from every member nodes present in the different cluster to the base station through optimal CHs. The following steps are implemented in HCSEHO algorithm for optimal cluster head selection:

Step 1: Initialization of WSN: Initialize the sensor network as per the constraints mention in parameter (Table I).

Step 2: Fitness assessment and global best declaration: The fitness assessment for each node is obtained with the help of objective function given in equation (3). Subsequently it is arranged in an ascending manner and the least solution of fitness is assigned to global best.

Step 3: Visibility check: The foraging of camels is impacted due to present of sand dunes. The visibility monitoring condition offers an accurate approach in the direction of the optimal result. If the condition for visibility checking is satisfied, the camel position is updated using camel algorithm. Else, it follows EHO algorithm for optimal cluster heads selection.

Step 4: Stop criterion: The procedure is recurrently executed for a defined number of search iterations.

Step 5: Data Transmission: Data transmission is initiated among the optimal CHs and the Sink node. This procedure is repeated for each iteration of information communication and continued until the iteration reaches a maximum number of rounds.

TABLE I. SiMULATION PARAMETERS OF HCSEHO ALGORITHM

\begin{tabular}{|l|l|}
\hline Parameter & Value \\
\hline Sensor search section $\left(\mathrm{m}^{2}\right)$ & $(200 * 200)$ \\
\hline Number of nodes $(\mathrm{n})$ & 100 \\
\hline Initial energy of a node( $\left.\mathrm{E}_{\mathrm{o}}\right)(\mathrm{J})$ & 0.5 \\
\hline Information length (I) (bits) & 4096 \\
\hline $\mathrm{E}_{\text {ec }}(\mathrm{nJ} / \mathrm{bit})$ & 70 \\
\hline $\mathrm{E}_{\text {amp }}\left(\mathrm{pJ} / \mathrm{bit} / \mathrm{m}^{2}\right)$ & 120 \\
\hline Energy data aggregation(nJ) & 5 \\
\hline $\begin{array}{l}\text { Number of Number of iterations for Data } \\
\text { Transmission }\left(\mathrm{r}_{\max }\right)\end{array}$ & 3000 \\
\hline Number of iterations for cluster head selection & 5 \\
\hline Total optimal Cluster Heads $(\mathrm{k})$ & 5 \\
\hline Visibility threshold & 0.1 \\
\hline Scale factor $\alpha, \beta)$ & $\alpha \in[0,1]$ \\
& $\delta \in[0,1]$ \\
\hline Particle location $\left[x_{\max }, x_{\min }\right]$ & {$[0,200]$} \\
\hline
\end{tabular}




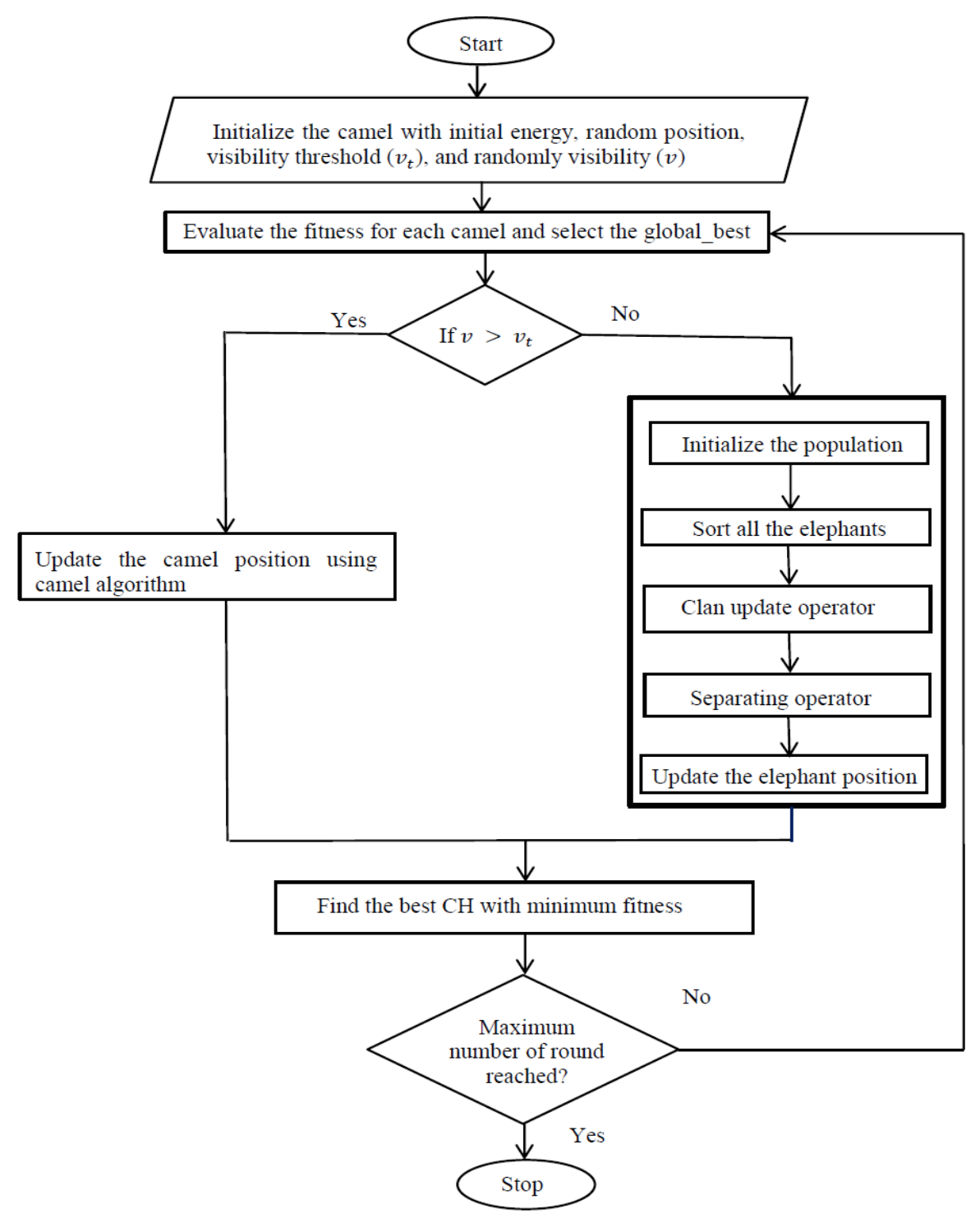

Fig. 1. Flowchart of the Proposed HCSEHO.

\section{RESUlTS AND DisCUSSION}

The simulation of the recommended HCSEHO method of CHS is executed in MATLAB R2018a plat form. The necessary parameters of the simulation taken into consideration for the offered technique are specified in Table I. The recommended HCSEHO is analyzed by comparing with the surviving CHS methods explicitly, Direct Transmission, LEACH [6], PSO [13], CA [5], and EHO [23].

Fig. 2 shows the comparative illustration of the performance of the different algorithms in terms of count of the alive nodes for an increasing number of rounds of data transmission. The first node in the WSN for the proposed HCSEHO is alive till the round number 2038, whereas, for the algorithms: Camel, EHO, PSO, LEACH, and DT, the nodes die at $1298,1602,1201,270$, and 44 , respectively. Half of the total number of nodes in the WSN for the proposed HGWSFO is alive till the round number 2147, whereas, for the algorithms: Camel, EHO, PSO, LEACH, and DT, the nodes die 1445,
1710, 1222, 370, and 74, respectively. All the nodes in the WSN for the proposed HGWSFO stay alive till the round number 2198, whereas, for the algorithms: Camel, EHO, PSO, LEACH, and DT, the nodes stay alive till 1512, 1736, 1229, 714 , and 315 rounds, respectively. The lifetime of the nodes for the proposed HCSEHO is $31.21 \%$ more than the Camel algorithm, 21.01\% more than the EHO algorithm, 44.08\% more than the PSO algorithm, 67.51\% more than the LEACH protocol, and $85.66 \%$ more than the DT.

Fig. 3 shows the comparative illustration of the performance of the different algorithms in terms of count of the dead nodes for an increasing number of rounds of data transmission. The first node in the WSN for the proposed HCSEHO dies at the round number 2038, whereas, for the algorithms: Camel, EHO, PSO, LEACH, and DT, the nodes die at $1298,1602,1201,270$, and 44 , respectively. Half of the total number of nodes in the WSN for the proposed HCSEHO die at the round number 2147, whereas, for the algorithms: Camel, EHO, PSO, LEACH, and DT, the nodes die at 1445, 1710, 
1222, 370, and 74, respectively. All the nodes in the WSN for the proposed HCSEHO die at the round number 2198, whereas, for the algorithms: Camel, EHO, PSO, LEACH, and DT, the nodes die at 1512, 1736, 1229, 714, and 315, respectively. The lifetime of the nodes for the proposed HCSEHO is $31.21 \%$ more than the Camel algorithm, 21.01\% more than the EHO algorithm, 44.08\% more than the PSO algorithm, 67.51\% more than the LEACH protocol, and $85.66 \%$ more than the DT.

Fig. 4 shows the comparative illustration of the performance of the different algorithms in terms of residual energy in $\mathrm{J}$ for an increasing number of iterations of information transmission. The residual energy in the WSN for all the algorithms at the initial round is $50 \mathrm{~J}$. When 1500 rounds are reached, the residual energy of the proposed HCSEHO is $15.49 \mathrm{~J}$, whereas, for the algorithms: Camel and EHO, the residual energies are $0.2251 \mathrm{~J}$ and $2.585 \mathrm{~J}$, respectively. The residual energies of PSO, LEACH, and DT become zero at the round number 1500 . The residual energy of the proposed HCSEHO at 1700th round is $10.94 \mathrm{~J}$. The residual energy of the proposed HCSEHO declines to zero by the round number 2198, while for the algorithms: Camel, EHO, PSO, LEACH, and DT, the residual energy declines at 1512, 1736, 1229, 714, and 315, respectively. The residual energy of proposed HCSEHO is 52.65\% more than the Camel algorithm, $39 \%$ more than the EHO algorithm, 67\% more than the PSO algorithm, 87.10\% more than the LEACH protocol, and $95.10 \%$ more than the DT. The following table.2 shows the comparative analysis of the performance of various CHS methods.

Fig. 5 shows the comparative illustration of the performance of the different algorithms in terms of throughput obtained in bps for an increasing number of rounds of data transmission. The throughput in the WSN for all the algorithms at the initial round is 409600 bps. When 1700 rounds are reached, the throughput of the proposed HCSEHO and EHO are 409600 bps and 282653 bps, whereas, for the algorithms: Camel, PSO, LEACH, and DT the throughput is 0 bps. The throughput of the proposed HCSEHO declines to zero at the round number 2198, while the algorithms: Camel, EHO, PSO, LEACH, and DT, declines at 1512, 1736, 1229, 714, and 315, respectively. The throughput lifetime of the proposed HCSEHO is 32.69\% more than the Camel algorithm, 20.35\% more than the EHO algorithm, 43\% more than the PSO algorithm, 83\% more than the LEACH protocol, and 95\% more than the DT.

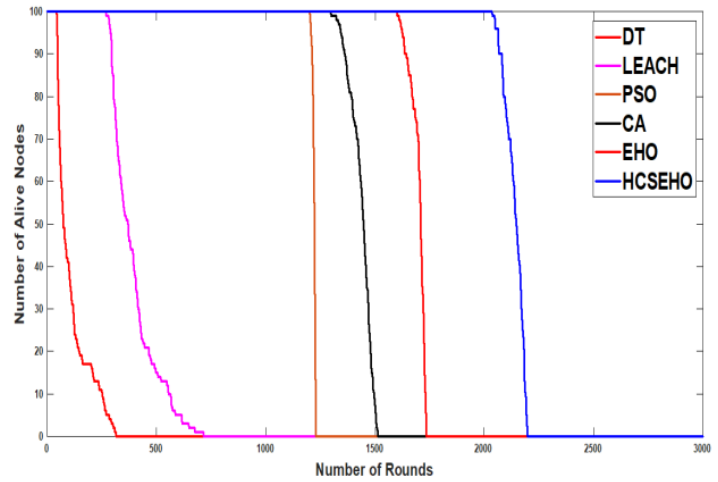

Fig. 2. Comparison of Alive Nodes Obtained for different CHS Algorithms.

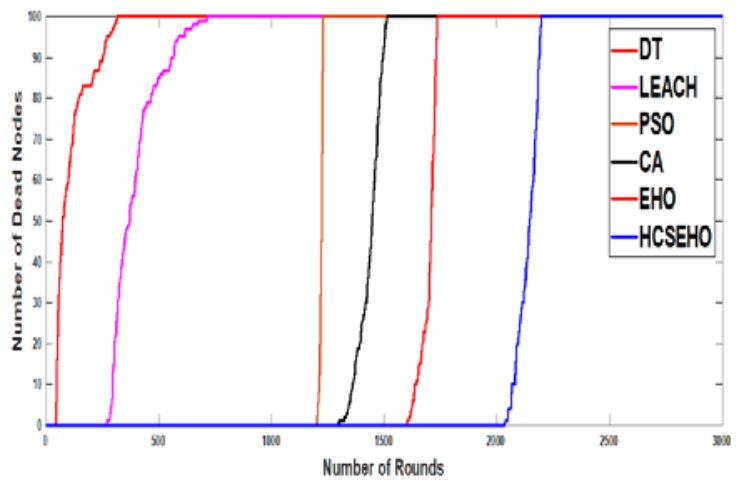

Fig. 3. Comparison of Dead Nodes Obtained for different CHS Algorithms.

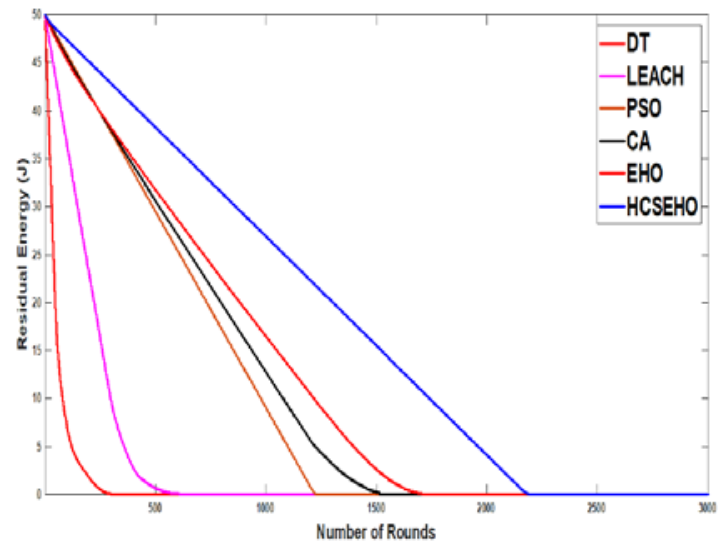

Fig. 4. Comparison of Residual Energy Obtained for different CHS Algorithms.

TABLE II. TABLE OF COMPARISON

\begin{tabular}{|c|c|c|c|c|c|c|c|c|c|}
\hline \multirow[b]{2}{*}{ Algorithm } & \multicolumn{3}{|c|}{ Dead Nodes (Rounds) } & \multicolumn{3}{|c|}{ Residual Energy (J) } & \multicolumn{3}{|c|}{ Throughput (bits/round) } \\
\hline & FND & HND & LND & $\begin{array}{l}\text { After } 1000 \\
\text { rounds }\end{array}$ & $\begin{array}{l}\text { After } 1500 \\
\text { rounds }\end{array}$ & $\begin{array}{l}\text { After } 1700 \\
\text { rounds }\end{array}$ & $\begin{array}{l}\text { After } 1000 \\
\text { rounds }\end{array}$ & $\begin{array}{l}\text { After } 1500 \\
\text { rounds }\end{array}$ & $\begin{array}{l}\text { After } 1700 \\
\text { rounds }\end{array}$ \\
\hline DT & 44 & 74 & 315 & 0 & 0 & 0 & 0 & 0 & 0 \\
\hline LEACH & 270 & 370 & 714 & 0 & 0 & 0 & 0 & 0 & 0 \\
\hline PSO & 1201 & 1222 & 1229 & 9.008 & 0 & 0 & 409600 & 0 & 0 \\
\hline CA & 1298 & 1445 & 1512 & 12.73 & 0.2251 & 0 & 409600 & 28675 & 0 \\
\hline EHO & 1602 & 1710 & 1736 & 16.45 & 2.585 & 0.0829 & 409600 & 409600 & 282653 \\
\hline Proposed HCSEHO & 2038 & 2147 & 2198 & 26.88 & 15.49 & 10.94 & 409600 & 409600 & 409600 \\
\hline
\end{tabular}




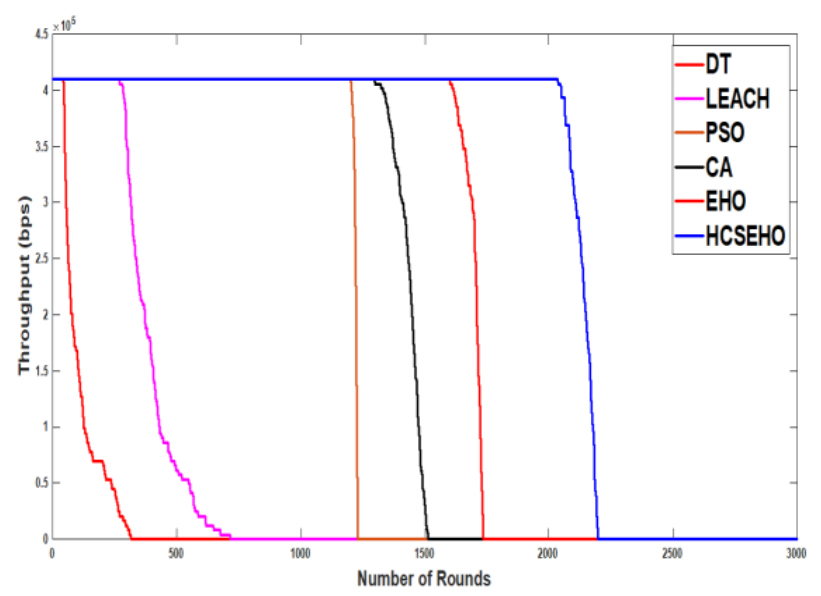

Fig. 5. Comparison of Throughputs Obtained for different CHS Algorithms.

\section{CONCLUSIONS}

This research methodology suggests HCSEHO algorithm for energy efficient cluster head selection in WSN by means of integrating two meta-heuristic algorithms explicitly, Camel and Elephant Herding Optimization. The energy consumption and separation distance are considered as the fitness function for selecting optimal CHs. The visibility monitoring condition of the camel algorithm enhances the efficiency of exploitation, whereas the exploration inefficiency of Camel algorithm is compensated in an optimal way by the EHO algorithm. The superior performance of the EHO replaces the random occurrence of the camel algorithm under the clan and separator operator. However, there is still an inadequacy in the EHO algorithm with respect to shortfall of exploitation towards an optimal convergence. The proposed HCSEHO is developed by integrating EHO algorithm with a high search efficient optimization algorithm called camel. The proposed HCSEHO is validated by comparing its performance with various other existing CHS algorithms in terms of throughput, residual energy, alive nodes, and dead nodes. It is found that the lifetime of the WSN guided by the proposed HCSEHO CHS shows $21.01 \%, 31.21 \%, 44.08 \%, 67.51 \%$, and $85.66 \%$, enhancement when compared to EHO, CA, PSO, LEACH, and DT methods, respectively.

\section{FUTURE WORK}

In the future, this work can be extended by conducting experiments for various types of sensor nodes.

\section{REFERENCES}

[1] I. F. Akyildiz, Y. Sankarasubramaniam, and E. Cayirci, "A survey on sensor networks”, IEEE Communications magazine, 2002, 40(8), pp. 102-114.

[2] A. Lindgren, A. Doria, and O. Schelén, Probabilistic routing in intermittently connected networks. In ACM International Symposium on Mobile Ad Hoc Networking and Computing, MobiHoc 2003: 01/06/2003-03/06/2003.

[3] Z. Jiang, and A. Wei. "An energy balanced algorithm of LEACH protocol in WSN". International Journal of Computer Science Issues (IJCSI), 2013, 10(1), pp. 354.
[4] S. Mirjalili, S.M Mirjalili, and Andrew Lewis, "Grey Wolf Optimizer”, Advances in Engineering Software, 2014, (69), pp.46-61.

[5] S. A. Ramzy, F. M. Alnahwi and S. A. Abdulkareem, "A Modified Camel Traveling Behaviour Algorithm for Engineering Applications", Australian Journal of Electrical and Electronics Engineering, 2019.

[6] W. B. Heinzelman, A. P. Chandrakasan, and H. Balakrishnan, "An application-specific protocol architecture for wireless microsensor networks". IEEE Transactions on wireless communications, 2002, 1(4), pp. 660-670.

[7] O. Younis, and S. Fahmy, “ HEED a hybrid, energy-efficient, distributed clustering approach for ad hoc sensor networks”, IEEE Transactions on mobile computing, 2004, (4), pp.366-379.

[8] N. Lavanya, and T. Shankar, "A Review on Energy-Efficient Scheduling Mechanisms in Wireless Sensor Networks". Indian Journal of Science and Technology, 2016, 9(32).

[9] S. Hussain, A. W. Matin, and O. Islam, "Genetic algorithm for hierarchical wireless sensor networks". Journal of Networks, 2007, 2(5), pp. 87-97.

[10] J. Rejina Parvin, and C. Vasanthanayaki, "Particle swarm optimizationbased clustering by preventing residual nodes in wireless sensor networks”. IEEE sensors journal , 2015, 15(8), pp.4264-4274.

[11] P. S. Rao, P. K. Jana, and H. Banka, "A particle swarm optimization based energy efficient cluster head selection algorithm for wireless sensor networks". Wireless networks , 2017 , 23(7), pp.2005-2020.

[12] Z. Wei-Guo, Y. Shao-Pu, L. Kui, and W. Li-Ying, "Gravitational search algorithm for node localization in wireless sensor network”, Information Technology Journal, 2016, 12(20), pp.5806-5811.

[13] C. Vimalarani, R. Subramanian, and S. N. Sivanandam, "An enhanced PSO-based clustering energy optimization algorithm for wireless sensor network", The Scientific World Journal, 2016.

[14] R. Kumar, and D. Kumar, "Multi-objective fractional artificial bee colony algorithm to energy aware routing protocol in wireless sensor network. Wireless Networks”, 2016, 22(5), pp.1461-1474.

[15] Z. Ye, and H. Mohamadian, "Adaptive clustering based dynamic routing of wireless sensor networks via generalized ant colony optimization". Ieri Procedia, 2014,10, pp.2-10.

[16] P. Lalwani, S. Das, H. Banka, and C. Kumar, "CRHS: clustering and routing in wireless sensor networks using harmony search algorithm", Neural Computing and Applications, 2018, 30(2), pp.639-659.

[17] X-S Yang, Flower pollination algorithm for global optimization.In: International conference on unconventional computing and natural computation. Springer, Berlin, 2012, pp 240-249.

[18] N. Lavanya, and T. Shankar, "Energy optimization in wireless sensor network using NSGA-II”, ARPN Journal of Engineering and Applied Sciences, 2006, 12(23).

[19] A. Genta, D. K. Lobiyal, and J. H. Abawajy, "Energy Efficient Multipath Routing Algorithm for Wireless Multimedia Sensor Network”. Sensors, 2019, 19(17),pp. 3642.

[20] Y.H. Niharika, and C. Agrawal, "A Review of Enhancing Energy Efficiency of Wireless Sensor Network", International Journal of Electrical, Electronics and Computer Engineering, 2019,pp.46-49.

[21] T. Shankar, S. Shanmugavel, and A. Rajesh, "Hybrid HSA and PSO algorithm for energy efficient cluster head selection in wireless sensor networks", Swarm and Evolutionary Computation ,2016, 30, pp.1-10.

[22] N. Lavanya, and T. Shankar, "Energy Efficient Cluster Head Selection Using Hybrid Squirrel Harmony Search Algorithm in WSN", International Journal of Advanced Computer Science and Applications, 2019,10(12).

[23] Gai-Ge Wang, Suash Deb, and Leandro dos S. Coelho, "Elephant Herding Optimization”, 2015, 3rd International Symposium on Computational and Business Intelligence.

[24] A. E. Mostafa, Ragab A. El Sehiemy, Yasser I. Rashwan , X.Z. Gao, "On the performance improvement of elephant herding optimization algorithm”, Knowledge-Based Systems , 2019,166, pp.58-70. 